AUTORES:

Cristiane A. de Farias

Bruno Rufino da Silva

Pedro Pinheiro Paes ${ }^{1}$

Departamento de Educação Física da Universidade Federal de Pernambuco, Brasil

\section{Avaliação da coordenação \\ motora em crianças \\ de 9 a 10 anos}

\section{PALAVRAS CHAVE:}

Coordenação motora. Avaliação. Criança

\title{
RESUMO
}

O professor de Educação Física tem sua atuação desafiada pelas diferenças biológicas e maturacionais dos seus alunos, sendo a coordenação motora é uma das capacidades físicas que apresenta a maior variação em crianças com idades semelhantes. Foi objetivo deste estudo identificar insuficiência no desenvolvimento da coordenação motora fina e grossa, relacionando com o gênero e a disponibilidade de áreas para essas crianças brincarem. Participaram deste estudo, 24 crianças, com idade média de $9.65 \pm 0.49$, de ambos os gêneros do 5 a ano do ensino fundamental de uma escola particular do Recife. A pesquisa foi de natureza observacional transversal, com a aplicação de três testes para avaliar a coordenação motora fina e grossa. Verificou-se que $70.83 \%$ das crianças avaliadas apresentaram coordenação motora fina bem desenvolvida, apresentando déficit no desenvolvimento da coordenação motora grossa, com 56.34\%, abaixo do nível satisfatório para a atividade testada. Observa-se que a diferença entre gênero e idade foi insignificante, devido a semelhança etária, como também não houve significância quando comparado com quem tinha uma área para brincar. Os resultados sugerem que as crianças praticam mais atividades de coordenação motora fina e em ambientes fechados em detrimento de atividades ao ar livre.

Evaluation of motor coordination

\section{ABSTRACT}

The professional of Physical Education has challenged his action because the biological and maturational differences of their students. The motor aspect, coordination is one of the physical abilities that exhibit strong variability in children with similar ages. OBJECTIVE: To identify failure in the development of fine and gross motor coordination, relating to gender and availability of these areas for children to play. METHODS: The study included 24 children with a mean age of $9.65 \pm 0.49$, of both genders enrolled in the 5 th year of elementary school, a private school in Recife. The research was a cross-sectional nature, with the application of three tests to assess fine motor coordination and thick. RESULTS: $70.83 \%$ of the children had well-developed fine motor coordination, presenting lost of the development of gross motor coordination, with 56.34 $\%$, below the satisfactory level for the tested activity. It is observed that the difference between gender and age was negligible, due to the proximity between the age groups. There was also no significance as the performance compared to those who had a play area. The results suggest that more children practice fine motor activities and indoor activities over outdoor environments.

KEY WORDS:

Motor coordination. Assessment. Child. 


\section{INTRODUÇÃo}

O professor de Educação Física, que atua no Ensino Fundamental, enfrenta muitas dificuldades no seu dia-a-dia, que implica diretamente no processo de ensino e aprendizagem, independentemente do ano letivo ou idade. Um desses desafios é representado pelas diferenças individuais entre cada criança, que pode variar muito nos aspectos de crescimento, desenvolvimento, maturação, habilidades sociais, cognitivas e motoras, mesmo em uma turma com faixa etária semelhante, fator que exige do profissional uma habilidade adicional para enfrentar essa realidade ${ }^{(13)}$

Atualmente, grande quantidade de crianças apresenta problemas no desenvolvimento motor, em decorrência do tempo que as mesmas passam sentadas em carteiras escolares ou em frente ao computador, diminuindo consequentemente o tempo disponível para se movimentar ${ }^{(5)}$

Segundo Krebs ${ }^{(7)}$, é no movimento que o desenvolvimento da criança se mostra de forma mais expressiva, sendo a principal base de interação e atuação da mesma com o meio físico e social. Logo, a função do professor de Educação Física é fazer atividades que possam ajudar no desenvolvimento motor da criança, incluindo, noções de direção, percepção espacial e temporal, flexibilidade, velocidade, coordenação motora, entre outras.

Para Gallahue e Ozmun (2), a coordenação motora é a eficiência na precisão nos movimentos do corpo, especialmente dos músculos e das articulações. Para Negrine ${ }^{(11)}$ a coordenação motora é a harmonização de uma sequencia de movimento, que presume a maturação do sistema nervoso. Ambos os autores dividem a coordenação motora em: coordenação motora grossa ou geral, que permite ao individuo equilibrar o corpo, utilizando os grandes músculos de forma mais produtiva e a coordenação motora fina, que permite utilizar pequenos músculos, para melhor manuseio de objetos e movimentos, de uma forma mais refinada.

Segundo Bessa e Pereira ${ }^{(s / n)}$, o desenvolvimento da habilidade de coordenação motora acontece principalmente nos seis primeiros anos de vida e o atraso no seu progresso pode afetar a criança, promovendo lentidão na realização dos movimentos e dificuldade na relação do próprio corpo com o meio. 0 déficit na coordenação motora durante a infância é frequente e pode interferir no seu desempenho escolar e social. Esses desvios no desenvolvimento motor são detectados facilmente nos movimentos instáveis dessas crianças Quando analisada insuficiência dessa habilidade, devem-se explorar novas práticas para estimular sua melhora, como é o caso da atividade física, que estimula o cérebro da criança, para o melhor controle de seus movimentos.

\section{OBJETIVOS}

O objeto deste estudo foi identificar a presença de insuficiência de desenvolvimento coordenativo em alunos da 5 a série do ensino fundamental I, na faixa etária entre 9 a 10 anos. Especificamente este estudo procura contemplar:

- Detectar o percentual de crianças que apresentam deficiências coordenativas, na amostra estudada;

- Identificar possíveis diferenças das habilidades coordenativas entre os gêneros:

- Relacionar as dificuldades de coordenação motora com a disponibilidade de áreas e/ou espaços de lazer na residência, ou adjacências (ruas, parques, clubes, etc).

\section{MÉTODO}

presente estudo é de natureza observacional, com corte transversal. Foi realizado com estudantes de uma escola particular de ensino regular, localizada no bairro de Mustardinha, na cidade do Recife. Participaram do estudo 24 crianças, de ambos os gêneros, com idade média de 9,65 $\pm 0,49$ anos, estudantes da 5 a série do ensino fundamental I, devidamente matriculadas e frequentes na unidade escolar investigada. Destes, 17 alunos eram do gênero feminino (70,83\%) e sete estudantes do gênero masculino $(29,17 \%)$.

Todos os pais e responsáveis pelos alunos que participaram do estudo assinaram um Termo de Consentimento Livre e Esclarecido, onde estava explicito o objetivo da pesquisa, o anonimato das pessoas envolvidas e quais testes as crianças seriam submetidas, seus riscos e benefícios, assim como a informação que a participação no estudo era de natureza facultativa.

Foi aplicado um questionário, com perguntas abertas, para identificação dos dados individuais dos alunos, incluindo variáveis como: idade, gênero, série, etc. Neste questionário, quatro perguntas foram voltadas para a análise da área física que a criança tinha para a prática de lazer na sua residência e/ou em áreas adjacentes, além do tipo de atividade que a criança normalmente praticava.

Para avaliar a habilidade de coordenação motora foram utilizados três testes. 0 primeiro (Teste 01) objetivava a avaliação da coordenação da motora fina, no qual a criança tinha que tocar dois alvos, de maneira simultânea, com duas canetas, dentro de vários quadrados impressos em uma de folha de papel A4, com área $10 \mathrm{~mm}^{2}$ e 0 alvo de $5 \mathrm{~mm}$ de diâmetro, localizado no centro de cada quadrado. Cada caneta deveria está acomodada em uma das mãos (mão direita 'a' e esquerda 'b') e os alvos deveriam ser tocados ao mesmo tempo. Cada criança executava três tentativas e poderia atingir os seguintes resultados: 
-0 ponto (não consegue tocar a ponta de nenhum dos lápis dentro do quadrado);

-1 ponto (consegue tocar a ponta de apenas um dos lápis dentro do quadrado e longe do alvo);

-2 pontos (consegue tocar as pontas dos dois lápis dentro do quadrado e fora do alvo);

-3 pontos (consegue tocar as pontas dos dois lápis um dentro do quadrado e um dentro do alvo);

-4 pontos (consegue tocar as pontas dos dois lápis no do alvo dentro do quadrado).

Para avaliar a coordenação motora grossa, foram aplicados dois testes, ambos executados através da atividade de pular corda, sendo que no primeiro teste (Teste 02) a própria criança batia a corda individualmente e o segundo teste (Teste 03) a corda era acionada por dois voluntários e a criança pulava a mesma. Os resultados para ambos os testes (02 e 03) eram feitos da seguinte forma:

- 0 ponto (não consegue realizar um ciclo completo da corda e/ou do salto):

-1 ponto (consegue realizar apenas um ciclo completo do pular corda);

-2 pontos (saltos sem "padrão temporal", não ajusta o salto à frequência da corda)

- 3 pontos (saltos com "padrão temporal", porém sem postura, sem estabilidade, com muita movimentação de pernas e braços);

-4 pontos (saltos com "padrão temporal", com postura, com estabilidade e movimento harmônico).

Os três testes utilizados neste estudo foram baseados em estudo semelhante desenvolvido por Pellegrini et al ${ }^{(13)}$, sendo que o teste 01, que avalia a coordenação motora fina, foi adaptado do teste original e criado um critério de avaliação previamente para esse estudo. Já nos testes 02 e 03 foi preservada a sua configuração e avaliação original.

As crianças foram avaliadas individualmente, em dois dias distintos, no período da manhã e tarde, com três tentativas para cada teste. Para efeito de registro dos dados foi computado o melhor desempenho entre as três tentativas, associado a uma pontuação que refletia a qualidade de cada uma das execuções.

Foram utilizadas técnicas estatísticas descritivas para a caracterização da amostra. Foi utilizado dois testes não paramétricos de Qui-quadrado (Chi-Square) e o Teste Exato de Fisher para as comparações entre grupos. Para o cálculo da intensidade de uma possível associação entre dois fatores/variáveis, utilizou-se o Coeficiente de Correlação de Pearson e Coeficiente de associação de Spearman. Foi considerando como nível de significância $p<0,05$.

\section{RESULTADOS E DISCUSSÃO}

CARACTERIZAÇÃO DA AMOSTRA ESTUDADA

(QUESTIONÁRIO DE IDENTIFICAÇÃO):

A pesquisa foi realizada com 24 crianças, de ambos os gêneros. Destes, houve predominância do gênero feminino com 70,8\%, (17) e apenas $29,2 \%$ do gênero masculino (7). (Tabela 1)

TABELA 1 - Distribuição da amostra por gênero.

\begin{tabular}{ccc}
\hline & QUANTIDADE & PERCENTUAL (\%) \\
\hline FEMININO & 17 & 70,8 \\
\hline MASCULINO & 7 & 29,2 \\
\hline TOTAL & 24 & 100,0 \\
\hline
\end{tabular}

Entre as crianças avaliadas ( $24=100 \%$ ), a média de idade foi $9,65 \pm 0,49$. Quando distribuído por gênero, o feminino (17) obteve média igual a 9,59 $\pm 0,51$ e o masculino (7), a média foi de $9,71 \pm 0,49$.

Ao que diz respeito a uma área para brincar, observou-se que a maioria das crianças $75,0 \%$ (18), possuía uma área disponível em sua casa para brincar. Porém, 68,8\% (11) mesmo assim brincavam nas ruas. Não foi encontrada uma associação entre os gêneros e as crianças possuírem uma área para brincar em casa, os dois gêneros apresentaram na sua maioria, como tendo uma área disponível para brincar (Tabela 2).

TABELA 2 - Relação das crianças por gênero x área para brincar x onde brinca

\begin{tabular}{|c|c|c|c|c|c|}
\hline \multirow{2}{*}{ SEXO } & & & \multicolumn{2}{|c|}{ TEM ÁREA PARA BRINCAR? } & \multirow[t]{2}{*}{ TOTAL } \\
\hline & & & SIM & NÃO & \\
\hline \multirow{3}{*}{ FEMININO } & \multirow{2}{*}{ ONDE BRINCA? } & EMCASA & $85,7(6)$ & $14,3(1)$ & $100(7)$ \\
\hline & & NA RUA & $70,0(7)$ & $30,0(3)$ & $100(10)$ \\
\hline & TOTAL & & $76,5(13)$ & $23,5(04)$ & $100(17)$ \\
\hline \multirow{3}{*}{ MASCULINO } & \multirow{2}{*}{ ONDE BRINCA? } & EMCASA & $100(1)$ & $0,0(0)$ & $100(1)$ \\
\hline & & NA RUA & $66,7(4)$ & $33,3(2)$ & $100(6)$ \\
\hline & TOTAL & & $71,4(5)$ & $28,6(2)$ & 100 (7) \\
\hline \multirow{3}{*}{ TOTAL } & \multirow{2}{*}{ ONDE BRINCA? } & EM CASA & $87,5(7)$ & $12,5(1)$ & $100(8)$ \\
\hline & & NA RUA & $68,8(11)$ & $31,2(6)$ & $100(17)$ \\
\hline & TOTAL & & $75,0(18)$ & $25,0(6)$ & $100(24)$ \\
\hline
\end{tabular}

$887-\operatorname{RPCD} 14$ (S1.A) 
A atividade de maior prevalência na infância é brincar. Collet et al ${ }^{(s / n)}$ afirmam que deve-se esperar melhor desempenho na coordenação motora das crianças que são mais ativas. Não havendo uma área adequada para brincar, as crianças são obrigadas a brincar em salas e quartos apertados, limitando dessa forma suas atividades e movimentos. Com isso as brincadeiras tradicionais como pula-corda, pega, amarelinha, entre outras estão sendo substituídas por atividades mais modernas e cada vez mais sedentárias, como videogame, computador, televisões, entre outras.

Quando perguntadas qual a brincadeira que as crianças mais gostavam, do total de crianças do sexo feminino $(17=100 \%)$ a maioria preferem brincar de "Pular corda" $(47,1 \%)$ e "Faz de conta" (17,6\%). Já entre o gênero masculino $(7=100 \%)$, afirmaram que preferem brincar de "futebol" $(71,4 \%)$ e de "pega pegou" $(28,6 \%)$. Observando-se assim, diferença estatisticamente significativa para $p \leq 0,05$, nos resultados obtidos quanto ao tipo de brincadeiras que as crianças mais gostam comparado ao gênero.

TABELA 3 - Distribuição por gênero e principal brincadeira

\begin{tabular}{|c|c|c|c|}
\hline \multirow{2}{*}{ PRINCIPAL BRINCADEIRA } & \multicolumn{2}{|c|}{ GÊNERO } & \multirow{2}{*}{ TOTAL } \\
\hline & FEMININO & MASCULINO & \\
\hline BONECA & $5,9(1)$ & $0,0(0)$ & 4,2 (1) \\
\hline FAZ DE CONTA & $17,6(3)$ & $0,0(0)$ & $12,5(3)$ \\
\hline FUTEBOL & $0,0(0)$ & $71,4(5)$ & $20,8(5)$ \\
\hline PAREDÃO & $5,9(1)$ & $0,0(0)$ & 4,2 (1) \\
\hline PEGA-PEGOU & $5,9(1)$ & $28,6(2)$ & $12,5(3)$ \\
\hline PULA ELÁSTICO & $5,9(1)$ & $0,0(0)$ & $4,2(1)$ \\
\hline PULA CORDA & $47,8(8)$ & $0,0(0)$ & $33,3(8)$ \\
\hline QUEIMADO & 5,9 (1) & $0,0(0)$ & 4,2 (1) \\
\hline VÔLEI & 5,9 (1) & $0,0(0)$ & 4,2 (1) \\
\hline TOTAL & $100(17)$ & $100(7)$ & $100(24)$ \\
\hline
\end{tabular}

Gallahue e Ozmun (2) afirmam que as diferenças de interesses entre meninas e meninos começam a se desenvolver justamente nesse período, pode-se constatar esse comportamento, nos tipos de brincadeiras escolhida por ambos (Tabela 3).
Silva ${ }^{(15)}$ diz que as brincadeiras oferecem às crianças a oportunidade de ampliar o desenvolvimento de seus aspectos motores, cognitivos e sociais. A autora destaca ainda que as brincadeiras tenham uma missão que vai além da ludicidade, é um momento educativo que enaltece o trabalho em grupo, aspectos psicológicos, habilidades motoras e melhora o desempenho em ambientes sociais.

Referente as brincadeiras de correr, saltar, subir em arvores e muros, a minoria das crianças, $45,8 \%$, responderam sim. Destas $35,3 \%$ são meninas e $71,4 \%$ são meninos. 0 percentual de crianças que relatou não se utilizar desse tipo de atividades chegou a 54,2\%, (Figura 7).

TABELA 4 - Distribuição das crianças por gênero e se brinca de correr, saltar e escalar

\begin{tabular}{cccc}
\hline \multirow{2}{*}{ SEXO } & \multicolumn{2}{c}{ BRINCA DE CORRER, SALTAR E ESCALAR? } & \multirow{2}{*}{ TOTAL } \\
\cline { 2 - 3 } & SIM & NÃO & \\
\hline FEMININO & $35,3(6)$ & $64,7(11)$ & $100(17)$ \\
\hline MASCULINO & $71,4(5)$ & $28,6(2)$ & $100(7)$ \\
\hline TOTAL & $45,8(11)$ & $54,2(13)$ & $100(24)$ \\
\hline
\end{tabular}

Andar, correr, saltar, são habilidades que nesse período, de nove e 10 anos, devem esta bem definidas. Porém, as diferenças individuais modificam essa perspectiva em algumas crianças, se estendendo até o final desse período, onde a coordenação das mesmas deverá esta bem estabelecida ${ }^{(13)}$

A análise dos dados, tanto da coordenação motora grossa como da coordenação motora fina mostra que algumas crianças que participaram do presente estudo tiveram certo impasse em executar as tarefas requisitadas. No entanto, $83,3 \%$ dessas crianças estão com a coordenação motora fina bem desenvolvida (Tabela 5) e uma carência na coordenação motora grossa, onde em média $58,4 \%$ dos alunos ainda não conseguiram se desenvolver muito bem. 0 que pode apenas ser reflexos dos tempos atuais, onde as crianças se prendem por muito tempo em uma sala de aula ou em frente do computador, contemplando redes sociais e jogos. Segundo Gallahue e Ozmun ${ }^{(2)}$, as restrições ambientais atrasam o aprendizado perceptivo-motor das crianças.

TESTE 01 - COORDENAÇÃO MOTORA FINA

De uma forma geral, as crianças tiveram um bom desempenho no teste de coordenação motora fina, com 83,3\% delas atingindo o objetivo, que era tocar a ponta dos dois lápis dentro do alvo, perfazendo o total de $4 p t$ para atividade. Somente $16,7 \%$ das crianças não conseguiram realizar esse movimento com precisão, com 12,5\% qualificadas com 3 pt e 4,2\% com 2 pt, de acordo com os critérios de qualificação pré estabelecidos para este teste (Tabela 4). 
Segundo Negrine ${ }^{(11)}$, a falta sinergia dos músculos, agonistas e antagonistas podem ser o principal motivo da dificuldade nessa habilidade. Com o amadurecimento de estruturas responsáveis pela sinergia da musculatura no cerebelo, vai se tornando mais definido os movimentos.

TESTE 03 - COORDENAÇÃO MOTORA GROSSA

0 desempenho dessas crianças melhorou quando os alunos realizaram o teste 03 , Nesse caso, $45,8 \%$ dos alunos, teve um bom desempenho, executando os saltos com bom padrão temporal, boa postura, estabilidade do corpo e movimento harmônico; $25,0 \%$ dos mesmos executaram os saltos sem ter uma boa estabilidade do corpo; $29,2 \%$ não conseguiram ajustar a frequência da corda aos seus saltos, sem conseguir realizar mais de dois saltos completos e nenhum dos alunos não conseguiu realizar no mínimo um ciclo completo do pular corda (Tabela 7).

TESTE 02 - COORDENAÇÃO MOTORA GROSSA:

As crianças não apresentaram um bom desempenho no Teste 02 . Apenas $37,5 \%$ dos alunos executaram os saltos com um ótimo padrão temporal, com boa postura, estabilidade do corpo e movimento harmônico; $12,5 \%$ dos mesmos executaram os saltos com um bom padrão temporal, porém sem postura adequada, sem conseguir estabilidade do corpo e com muita movimentação de pernas e braços; $16,7 \%$ não conseguiram ajustar o salto à frequência da corda, às vezes conseguindo não mais que dois ciclos completos da corda; $12,5 \%$ dos alunos conseguiram realizar apenas um ciclo completo do pular corda. E 20,8\% desses alunos não conseguiram realizar nem ao menos um ciclo completo da corda e/ou do salto, adquirindo pontuação 0 (Tabela 6).

TABELA 6 - Resultados do teste 02

\begin{tabular}{ccc}
\hline & QUANTIDADE & PERCENTUAL (\%) \\
\hline OPT & 5 & 20,8 \\
\hline I PT & 3 & 12,5 \\
\hline 2PT & 3 & 12,5 \\
\hline 3 PT & 4 & 16,7 \\
\hline 4 PT & 9 & 37,5 \\
\hline TOTAL & 24 & 100 \\
\hline
\end{tabular}

TABELA 7 - Resultados do teste 03

\begin{tabular}{ccc}
\hline & QUANTIDADE & PERCENTUAL (\%) \\
\hline OPT & 0 & 0,0 \\
\hline P PT & 0 & 0,0 \\
\hline 2 PT & 7 & 29,2 \\
\hline 3 PT & 6 & 25,0 \\
\hline 4 PT & 11 & 45,8 \\
\hline TOTAL & 24 & 100
\end{tabular}

Essa dificuldade na coordenação motora grossa ou geral encontrada nos Testes 02 e 03 pode ser observada no jeito de andar, nas atividades de lazer das crianças, no mau desempenho em esporte ou até na dificuldade de interagir com o meio.

Segundo Gallahue e $\mathrm{Ozmuz}{ }^{(2)}$, a criança nessa faixa etária se encontra numa fase entre a adolescência e a infância. É de natural nesse período um progresso lento e estável na composição corporal das crianças, principalmente na altura e no peso, e por uma evolução do arranjo no sistema sensorial e motor. A coordenação olho-mão e olho-pé são dificultados pelo tempo de reação das crianças, que são bastante lentas durante essa fase. 
DIFERENÇAS ENTRE OS GÊNEROS

Analisa-se nesse estudo a homogeneidade das amostras nos resultados dos três testes aplicados.

Não se encontraram diferenças estatisticamente significativas para p 0,05 , no desempenho das crianças no teste 01 , quando comparado por gênero. (Tabela 8 ).

TABELA 8 - Resultados do teste 01 comparado por gênero

\begin{tabular}{cccc}
\hline & FEMININO & MASCULINO & TOTAL \\
\hline OPT & $0,0(0)$ & $0,0(0)$ & $0,0(0)$ \\
\hline P PT & $0,0(0)$ & $0,0(0)$ & $0,0(0)$ \\
\hline 2PT & $0,0(0)$ & $14,3(1)$ & $4,2(1)$ \\
\hline 3 PT & $17,6(3)$ & $0,0(0)$ & $12,5(3)$ \\
\hline 4 PT & $82,4(14)$ & $85,7(6)$ & $83,3(20)$ \\
\hline TOTAL & $100(17)$ & $100(7)$ & $100(24)$
\end{tabular}

Não ocorreu associação significativa para $p \leq 0,05$, no desempenho das crianças no teste 02 quando comparado por gênero (Tabela 9).

TABELA 9 - Resultados do teste 02 comparado por gênero

\begin{tabular}{cccc}
\hline & FEMININO & MASCULINO & TOTAL \\
\hline O PT & $29,4(5)$ & $0,0(0)$ & $20,8(5)$ \\
\hline P PT & $11,8(2)$ & $14,3(1)$ & $12,5(3)$ \\
\hline 2 PT & $11,8(2)$ & $14,3(1)$ & $12,5(3)$ \\
\hline 3 PT & $11,8(2)$ & $28,6(2)$ & $16,7(4)$ \\
\hline 4 PT & $35,3(6)$ & $42,9(3)$ & $37,5(9)$ \\
\hline TOTAL & $100(17)$ & $100(7)$ & $100(24)$ \\
\hline
\end{tabular}

No teste 03 , também houve diferença significativa para $p \leq 0,05$, quanto ao desempenho das crianças comparado ao gênero.
TABELA 10 - Resultados do teste 03 comparado por gênero

\begin{tabular}{cccc}
\hline & FEMININO & MASCULINO & TOTAL \\
\hline OPT & $0,0(0)$ & $0,0(0)$ & $0,0(0)$ \\
\hline 1 PT & $0,0(0)$ & $0,0(0)$ & $0,0(0)$ \\
\hline 2 PT & $29,4(5)$ & $28,6(2)$ & $29,2(7)$ \\
\hline 3PT & $23,5(4)$ & $28,6(2)$ & $25,0(6)$ \\
\hline 4 PT & $47,1(8)$ & $42,9(3)$ & $45,8(11)$ \\
\hline TOTAL & $100(17)$ & $100(7)$ & $100(24)$ \\
\hline
\end{tabular}

COORDENAÇÃO MOTORA E DISPONIBILIDADE DE ÁREAS PARA O LAZER:

Observando o desempenho das crianças nos três testes que avaliava a coordenação motora relacionado com a disponibilidade de uma área para as mesmas brincarem, nota-se que há uma homogeneidade entre o desempenho das crianças, não havendo uma diferença relevante para $p \leq 0,05$, que possa interferir nos resultados dos testes (Tabela 11).

TABELA 11 - Distribuição das crianças por desempenho e área de brincar

\begin{tabular}{|c|c|c|c|c|}
\hline & \multirow{2}{*}{ DESEMPENHO } & \multicolumn{2}{|c|}{ ÁREA PARA BRINCAR } & \multirow{2}{*}{ TOTAL } \\
\hline & & SIM & NÃO & \\
\hline \multirow{5}{*}{ TESTE 01} & OPT & $0,0(0)$ & $0,0(0)$ & $0,0(0)$ \\
\hline & 1 PT & $0,0(0)$ & $0,0(0)$ & $0,0(0)$ \\
\hline & $2 \mathrm{PT}$ & $5,6(1)$ & $0,0(0)$ & 4,2 (1) \\
\hline & 3 РT & $16,7(3)$ & $0,0(0)$ & $12,5(3)$ \\
\hline & 4 PT & $77,8(14)$ & $100(6)$ & $83,3(20)$ \\
\hline \multirow[t]{3}{*}{ TOTAL } & & $100(18)$ & $100(6)$ & $100(24)$ \\
\hline & OPT & $22,2(4)$ & $16,7(1)$ & $20,8(5)$ \\
\hline & $1 \mathrm{PT}$ & 11,1 (2) & $16,7(1)$ & $12,5(3)$ \\
\hline \multirow[t]{3}{*}{ TESTE 02} & $2 \mathrm{PT}$ & 11,1 (2) & $16,7(1)$ & $12,5(3)$ \\
\hline & 3 PT & $16,7(3)$ & $16,7(1)$ & $16,7(4)$ \\
\hline & 4 PT & $38,9(7)$ & 33,1 (2) & 37,5 (9) \\
\hline
\end{tabular}

893 - RPCD 14 (S1.A) 


\begin{tabular}{|c|c|c|c|c|}
\hline & \multirow{2}{*}{ DESEMPENHO } & \multicolumn{2}{|c|}{ ÁREA PARA BRINCAR } & \multirow{2}{*}{ TOTAL } \\
\hline & & SIM & NÃO & \\
\hline \multirow[t]{3}{*}{ TOTAL } & & $100(18)$ & $100(6)$ & $100(24)$ \\
\hline & $0 P T$ & $0,0(0)$ & $0,0(0)$ & $0,0(0)$ \\
\hline & 1 PT & $0,0(0)$ & $0,0(0)$ & $0,0(0)$ \\
\hline \multirow[t]{3}{*}{ TESTE 3} & $2 \mathrm{PT}$ & $22,2(4)$ & $50,0(3)$ & $29,2(7)$ \\
\hline & 3 PT & $27,8(5)$ & $16,7(1)$ & $25,0(6)$ \\
\hline & 4 PT & 50,0 (9) & $33,3(2)$ & $45,8(11)$ \\
\hline TOTAL & & $100(18)$ & $100(6)$ & $100(24)$ \\
\hline
\end{tabular}

1. CORDAZZO, S. T. D.; VIEIRA, MAURO L. V. Caracterização de brincadeiras de crianças em idade escolar. Psicologia: reflexão e crítica, Porto Alegre, v. 21, n. 3, 2008. Disponivel em: <http://www.scielo.br/scielo. php?pid $=$ S0102- $79722008000300004 \&$ script $=$ sci arttext> Acessado em: 17 jul. 2013

2. GALLAHUE, D; OZMUN, J. Compreendendo o desenvolvimento motor: bebes crianças, adolescentes adultos. 3.ed, São Paulo: Phorte, 2005.

3. GONÇALVES, E. F. Perfil Psicomotor: Caracterização de Alunos da $3^{\circ} \mathrm{e} 4^{\circ}$ Séries do Ensino Fundamenta do Núcleo Cruz Vermelha-PEI, Programa Escolinha Integrada. EFDeportes.com: Revista Digital. Buenos Aires, Ano 13, n. 120, 2008. Disponivel em: <http http://www.efdeportes.com/efd120/perfil-psicomotor-caracterizacao-de-alunos-do-ensino-fundamental.htm> Acessado em 21 abr. 2013.

4. GORLA,J.I., ARAÚJO, P. F. Avaliação Motora em Educação Física Adaptada: Teste KTK para Deficientes Mentais. Editora Phorte, p. 54-56, 2007.

5. GUISELINI, MAURO, A. Tarefas Motoras para Crianças em idade Pré-escolar. São Paulo: CLR Baleiro, 1987. 6. HAYWOOD, K. M. Life span motor development. 2nd Edition. Champaign, IL, Human Kinetics Publishers, 1993. 7. KREBS. R. J. Desenvolvimento Infantil em Contexto. Florianópolis: Editora da UDESC, 2001.

8. LAKATOS, M, E; MARCONI, A, M. Metodologia Cientifica. 3.ed, São Paulo: Atlas, 2008

9. MAGALHÃES, L, C, et all. Estudo Comparativo Sobre o Desempenho Perceptual e Motorna Idade Escolar em Crianças Nascidas Pré-termo e a Termo. Arq. Neuropsiquiatr, 2003; 61 (2-A): 250-255.

10. NASCIMENTO, V. S; Leite, W. S; e Magalhães, L, C. Coordenação motora fina em crianças na idade escolar: demandas da sala de aula. Bireme, São Paulo, Agosto 2003. 11. NEGRINE. A. da S. A Coordenação Psicomotora e Suas Implicações. Porto Alegre, 1987

12. PAIM, C. C. M. Desenvolvimento Motor de crianças pré-escolares entre 5 e 6 anos. EFDeportes.com, Revista Digital. Buenos Aires, Ano 8, n.58, Mar, 2003. Disponivel em: <"http://www.efdeportes.com "www.efdeportes.com/efd58/5anos.htm> Acessado em: 21 abr. 2013
13. PELLEGRINI, A. M. et AL. Desenvolvendo a coordenação motora no ensino fundamental. Disponível em: <http://www.unesp.br/prograd/PDFNE2003/Desenvolvendo\%20a\%20coordenacao\%20motora.pdf $>$ Acessado em: 23 Jun. 2013

14. PELLEGRINI, A. M. 0 correr e o arremessar em crianças de 6 a 8 anos de idade In: IV Seminário Brasileiro de Pesquisa em Educação Física e Desporto. Anais do IV Seminário Brasileiro de Pesquisa em Educação Física e Desporto, Santa Maria, RS, 1985.

15. SILVA, K. C. M. Além dos muros escolares: Um resgate dos jogos tradicionais nas aulas de educação física. EFDeportes.com, Revista Digital.Buenos Aires, Ano 10, n 88, Setembro de 2005. Disponivel em: <"http:// www.efdeportes.com"www.efdeportes.com/efd88/ jogos.htm> Acessado em: 14 mar. 2013.

16. SILVEIRA, C. R. A. Avaliação Motora de Pré-Escolares: Relação Entre Idade Motora e Idade Cronológica. EFDeportes.com, Revista Digital. Buenos Aires, Ano 10, n 83, Abril de 2005. Disponível em: <"http:// www.efdeportes.com"www.efdeportes.com/efd83/ avalia.htm> Acessado em: 17 mar. 2013.

17. TUBINO, M. J. G. As Qualidades Físicas na Educação Física e Desporto. Rio de Janeiro, Fórum, 1997. 